\title{
The Arabidopsis pentatricopeptide repeat protein PDM1 is associated with the intergenic sequence of S11-rpoA for rpoA monocistronic RNA cleavage
}

\author{
YIN QianQian ${ }^{\dagger}$, CUI YongLan ${ }^{\dagger}$, ZHANG GuoRui, ZHANG HongDao, WANG XiaoMeng \& \\ YANG ZhongNan*
}

College of Life and Environmental Sciences, Shanghai Normal University, Shanghai 200234, China

Received April 16, 2012; accepted May 8, 2012; published online May 23, 2012

\begin{abstract}
The PDM1 gene encodes a pentatricopeptide repeat protein of the PLS subfamily. It is essential for $r p o A$ polycistronic processing in Arabidopsis. In this study, we performed functional analysis of PDM1 in rpoA monocistronic cleavage and chloroplast development. The $p d m 1$ mutants display an albino lethal phenotype with severe chloroplast development defects. When the construct of PDM1 fused with the GFP gene was introduced into Arabidopsis protoplasts, the GFP signal was exclusively observed in chloroplasts. This shows that PDM1 is localized to chloroplast. In the wild type, the rpoA transcript of about 990 nt is processed. In $p d m 1$, this transcript is absent. However, Western blot showed that the RpoA protein in $p d m 1$ is accumulated at a level approximately $1 / 3$ of the wild type. This suggests that certain other transcripts processed from L23-L2-S19-L22-S3-L16-L14S8-L36-S11-rpoA polycistronic precursor may be used as templates for protein translation. To determine whether PDM1 can bind to the rpoA pre-mRNA, we generated a transgenic Arabidopsis line with PDM1 fused to FLAG tag that was capable of complementing the $p d m 1-1$ mutant phenotype. RNA immunoprecipitation analysis showed that PDM1 is associated with $S 11-r p o A$ intergenic sequence. This indicates that PDM1 may bind to the S11-rpoA intergenic region to perform rpoA processing.
\end{abstract}

Arabidopsis, PDM1, RNA cleavage

Citation: Yin Q Q, Cui Y L, Zhang G R, et al. The Arabidopsis pentatricopeptide repeat protein PDM1 is associated with the intergenic sequence of S11-rpoA for rpoA monocistronic RNA cleavage. Chin Sci Bull, 2012, 57: 3452-3459, doi: 10.1007/s11434-012-5278-9

Chloroplasts are derived from endosymbiotic cyanobacteria [1]. Throughout the course of evolution, the chloroplast genome has retained its prokaryotic characteristics and taken on certain new characteristics of the eukaryotic genome. As in the genome of cyanobacteria, many of the chloroplast genes are organized in multiple transcription units that are transcribed into polycistronic pre-mRNAs, which are then mostly cleaved into monocistronic mRNAs [2]. However, the chloroplast genome has evolved elaborate posttranscriptional RNA processing steps as well as eukaryotes. These processing steps include RNA splicing, RNA editing and exonucleolytic trimming for the purpose of stabilization and translation.

$†$ These authors contributed equally to this work.

*Corresponding author (email: znyang@ shnu.edu.cn)
Recently, pentatricopeptide repeat proteins (PPRs) were reported to be involved in these RNA processing steps in chloroplasts. The PPR proteins are characterized by a degenerate motif of 35 amino acids that can be repeated up to 30 times within a single protein. Approximately half of the PPR proteins in Arabidopsis thaliana display tandem arrays of canonical repeats (or P-repeats). These proteins are known as P-type PPRs. Other family members, known as PLS subfamily, contain not only the standard 35 amino acid repeats, but also a shorter (S-repeats) and a longer version (L-repeats). The PLS subfamily proteins are further divided into PLS, E, E+ and DYW subgroups based on the presence of additional domains at the $\mathrm{C}$ terminus [3]. Numerous PPRs play roles in splicing [4-6]. Some of the PPR proteins may have an effect on both RNA stability and translation [7,8]. Fifteen 
PPRs have been found to have RNA-editing functions; they serve 22 of the 34 known editing sites in Arabidopsis [9].

PPR proteins are also reported to be involved in monocistronic transcripts cleavage from polycistronic ones. They include HCF152 [5] and CRR2 [10] in Arabidopsis and CRP1 [11] in maize. HCF152 is essential for endonucleolytic cleavage between $p s b H$ and petB. It binds certain areas of the pet $B$ transcripts. CRP1 is necessary for the processing of the monocistronic petD mRNA from the polycistronic precursor and for the translation of the chloroplast petA transcripts. It exhibits significant structural similarity to HCF152. CRR2 functions in the intergenic processing of chloroplast $r p s 7-n d h B$ dicistronic transcript. It is essential for $n d h B$ translation.

Plastid genes in higher plants are transcribed by plastid-encoded polymerase (PEP) and nucleus-encoded polymerase (NEP). Most plastid genes are transcribed by plastid-encoded polymerase (PEP). RpoA is a core subunit of PEP. In Arabidopsis thaliana, the monocistronic rpoA transcript of approximately 990 nucleotides is processed from the L23-L2-S19-L22-S3-L16-L14-S8-L36-S11-rpoA polycistron. The previous investigation shows that the PPR protein PDM1 is involved in the processing of the rpoA pre-mRNA in Arabidopsis thaliana [12]. In $p d m 1$, the rpoA transcript of 990nt was absent. However, the molecular mechanism for PDM1 to regulate the rpoA mRNA processing is not clear. In this study, we performed functional analysis of PDM1 in rpoA monocistronic cleavage and chloroplast development in Arabidopsis.

\section{Materials and methods}

\subsection{Plant material and growth conditions}

Arabidopsis mutant pdm1-4 (Landsberg erecta background) was screened using an EMS mutagenesis strategy. Prior to phenotypic analysis, pdml-4 was backcrossed to Landsberg erecta ecotype three times. The T-DNA insertion lines ( $\mathrm{Co}$ lumbia ecotype) were obtained from the Arabidopsis Biological Resource Center (ABRC; Ohio State University). Seeds were sown on vermiculite and MS medium agar plates according to the different experimental procedures after imbibition for $3 \mathrm{~d}$ at $4{ }^{\circ} \mathrm{C}$. Plants were grown under long-day conditions ( $16 \mathrm{~h}$ of light/ $8 \mathrm{~h}$ of dark) at a constant temperature of $22^{\circ} \mathrm{C}$.

\subsection{Phenotype characterization and microscopy}

Plants were photographed with a Nikon digital camera, Coolpix 4500 (Nikon, Japan). Small leaf segments were obtained from 2-week-old plants grown on MS medium agar plates added $2 \%$ sucrose. Transmission electron micrographs (TEMs) were obtained exactly as described [13]. The specimens were examined under transmission electron microscopy (Hitachi H7650; Hitachi, http: //www.hitachi.com).

\subsection{Molecular cloning of the PDM1 gene}

The presence of the T-DNA insertion in the mutant was validated using primers that specifically amplified a sequence of the T-DNA (F: 5'-AGATTATTGCTCGGGTAGAT-3'; R: 5'-GCTAATGGTAATGGTGCTAC-3'). Co-segregation of the T-DNA insertion site and the mutant phenotype were analyzed with AtLBb1.3 and the plant-specific primers ( $p d m$ l-1-LP: 5'-AAAGGCCATAAACTTGGAAGC-3'; pdm1-1-RP: 5'-TTCATGGACCGCTATGATCTC-3'; pdml-2-LP: 5'-TTCTTCTTTGCAATGGAATGG-3'; $p d m 1$ 2-RP: 5'-TGCACGTAAAGTGTTCGACAG-3'; $p d m 1$-3-LP: 5'-TGGAAATGTGTCACTAAGGCC-3'; pdml-3-RP: 5'AAACACGGTCACATGGACTTC-3'). For each of the mutant plant, PCR using AtLBb1.3 and specific RP primers amplified a specific DNA fragment. For the WT plants, PCR using paired LP and RP primers amplified longer specific DNA fragments. PCR with both primer pairs exhibited positive results for the heterozygous mutant plants.

Complementation experiment was performed as follows: a DNA fragment of $3834 \mathrm{bp}$, including an $1983 \mathrm{bp}$ upstream and $1851 \mathrm{bp}$ coding sequence without a termination codon of PDM1 was amplified and fused to a FLAG-tagged sequence (DYKDDDDK, nucleotides GACTACAAAGACGATGACGACAAG) using KOD plus polymerase (TAKARA, Japan) (CMP-F: 5'-CCATGGATACTGCTTCCAGTCATC-3'; CMPR: 5'-AGATGTCTCCAAGTAACAAGTTGC-3'). Following sequence verification, the fragment was cloned into the pCAMBIA1300 binary vector (CAMBIA; http://www. cambia.org.au), then the recombinant was introduced into heterozygous plants using the infiltration method by Agrobacterium strain LBA4404. The transformants were selected on PNS culture medium with $80 \mathrm{mg} \mathrm{L}^{-1}$ hygromycin, and screened for plants with a green phenotype and a homozygous background. The primer sets were used as follows: AtLB3/RP primers were used to validate the presence of a T-DNA insertion in pdml-1; genomic specific primers CI-F/CI-R (CI-F: 5'-TAGAAACACGGTCACATGG-3'; CIR: 5'-ATTTGGTGCTATTGTGGAA-3') were used to validate the homozygous ( $p d m l / p d m l)$ background.

\subsection{Subcellular localization of the PDM1 fused GFP protein}

For GFP fusion, the 201-bp sequence containing the plastid transit peptide encoding sequence and the full-length CDS lacking a stop codon were respectively cloned from the seedling cDNA of the wild type with the following primers: $P D M 1$-TP-F: 5'-ATGTTTTCGTTATCGTTAAT-3'; PDM1$T P$-R: 5'-TCCTTTGAAACCACTAAAAC-3'; PDM1-CDSF: 5'-ATGTTTTCGTTATCGTTAAT-3', PDM1-CDS-R: 5'AGATGTCTCCAAGTAACAAG- $3^{\prime}$. The two sequences respectively fused with $e G F P$ were cloned into the pMON530 binary vector. Transformation was performed as described above and the transformants were selected using $50 \mathrm{mg} / \mathrm{L}$ 
kanamycin. The GFP fluorescence of the transgenic plants was observed under Confocal Laser Scanning Microscopy (LSM 5 PASCAL; ZEISS, http://www.zeiss.com).

\subsection{Phylogenetic analysis}

The multiple sequence alignment of the full-length protein sequences was performed using the ClustalX tool. The phylogenetic tree was constructed and tested by MEGA3.1 based on the UPGMA method.

\subsection{Western blot analysis}

Total leaf proteins were extracted, fractionated by SDSPAGE and analyzed using immunoblotting, as described previously [14]. The anti-FLAG M2 monoclonal antibody was purchased from Sigma-Aldrich (http://www.sigmaaldrich.com), and the anti-RpoA polyclone antibody was raised against a synthetic peptide of RpoA by the GL Biochem company (http://www.glbiochem.com). The RpoA peptide was composed of the following amino acids: KCVESKRDSKRLYYGR. We identified the activity and specificity of the anti-RpoA antibodies as follows: The rpoA gene from Arabidopsis was recombined with pET-51b vector and expressed in E. coli Rosseta strain. Western blot was then performed to verify the anti-RpoA antibodies against the expressing RpoA peptide in E. coli Rosseta strain. The empty pET-51b was used as a negative control.

\subsection{RNA immunoprecipitation}

Arabidopsis seedlings ( $21 \mathrm{~d}$ old) were fixed with $1 \%$ formaldehyde. Then the chloroplast isolation was performed according to Ketcham et al. [15]. The chloroplast was used for immunoprecipitation of specific RNA-protein complexes according to the description of Terzil et al. [16]. The anti-FLAG M2 monoclonal antibody and Dynabeads ${ }^{\circledR}$ ProteinG were purchased from Sigma-Aldrich and Invitrogen respectively (Sigma, http://www.sigmaaldrich.com; Invitrogen, http://www.invitrogen.com). RNA was isolated, purified and reverse-transcribed according to Cao et al. [17]. For real-time PCR, $1 \mu \mathrm{L}$ of the cDNA was analyzed in a 20 $\mu \mathrm{L}$ reaction using the Quantitect SybrGreen PCR kit (Qiagen, http://www.qiagen.com) in an ABI7300 system (BioRad, http://www.bio-rad.com). Each realtime PCR reaction was performed in triplicate. Primers used in the analysis are listed in Table S1.

\section{Results}

\subsection{A point mutation in PDM1 leads to an albino phenotype}

To identify gene important for chloroplast development, an albino mutant was identified from a population of the Ara- bidopsis Landsberg erecta ecotype (Ler) mutagenized with ethyl methane sulphonate (Figure 1(a)). Genetic analysis revealed this phenotype resulted from a single recessive locus (data not shown). The mutated locus was mapped using an $\mathrm{F}_{2}$ population generated by crossing the heterozygote with the Arabidopsis Columbia ecotype (Col). For the firstpass mapping, a total of 20 insertion/deletion (In/Del) markers were used [18], and the mutated locus was linked to the In/Del marker FCA6 on chromosome 4. Fine mapping was carried out using a population of approximate 1800 albino progenies. The mutated locus was narrowed to a region of $49.6 \mathrm{~kb}$ between F28J12 and F28A21 on chromosome 4 (Figure 1(b)).

This region contains 13 putative genes, among which PDM1 (At4g18520) encodes a PPR protein localized to chloroplast. Sequence analysis showed that a TGG to TGA mutation occurred in the mutant which caused translation to end prematurely in $P D M 1$, resulting in a truncated form of 426 aa from a full length protein of 617 aa. We obtained three knockout lines for PDMI from the SIGnAL collection at the Arabidopsis Biological Resource Center (ABRC), pdml-1(SALK_034701), pdml-2 (CS835488) and pdml-3 (SALK_005288). The T-DNA insertions in PDM1 were confirmed by PCR analysis (Figure 1(c)). Semiquantitative RT-PCR analysis showed that the PDMI expression was not detectable in these mutants (Figure 1(d)). Allelism tests indicated that our mutant and these $p d m 1$ mutants belonged to the same locus mutation (data not shown). Therefore, our mutant is named $p d m 1-4$. As the four alleles exhibit a similar phenotype, $p d m l-1$ was chosen for further analysis.

To further investigate whether $P D M 1$ is responsible for the albino phenotype, we carried out a genetic complementation experiment. The PDM1 genomic fragment, including a 1983-bp sequence upstream from the initiation codon, the $3^{\prime}$ end of which lacks a termination codon, was fused to a $F L A G$-tagged sequence. This fused sequence was introduced into PDMl/pdml heterozygous plants through Agrobacterium tumefaciens-mediated transformation. Eight transgenic plants carrying the complementation construct were obtained. These transgenic lines show the same phenotype as the WT. PCR analysis confirmed that the genetic background of one transgenic line was $p d m 1 / p d m 1$. These results verified that $P D M I$ was responsible for the $p d m 1$ albino phenotype.

\subsection{Chloroplast development is defective in pdm 1-1 mutant}

When grown in plant soil, the pdm1-1 mutant displayed albino cotyledons. It could not survive more than $10 \mathrm{~d}$ after germination. When grown in MS medium containing 2\% sucrose, it produced two to four etiolated true leaves. However, it died shortly after developing the second pair of true leaves (Figure 1(a)). To understand the chloroplast developmental defects in $p d m 1-1$, the morphology and ultrastructure 
(a)

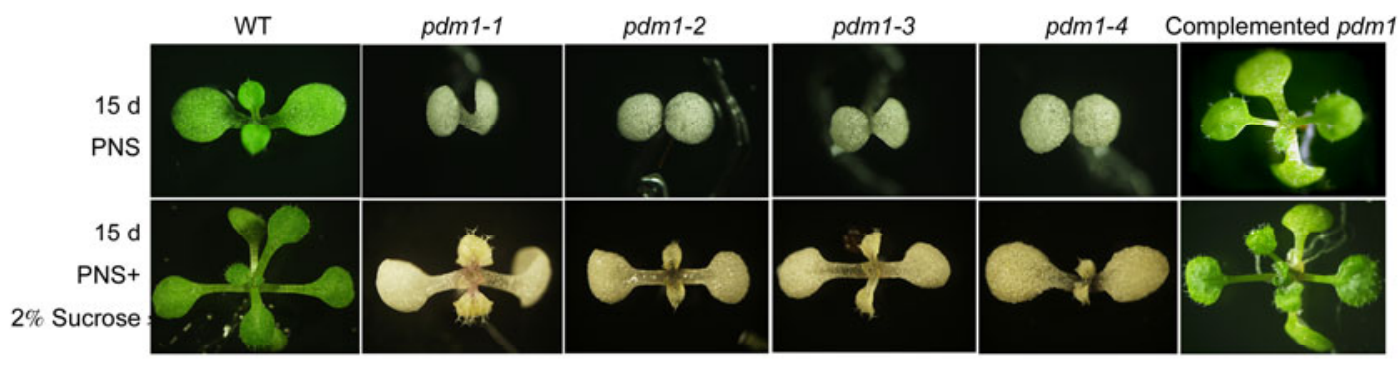

(b)

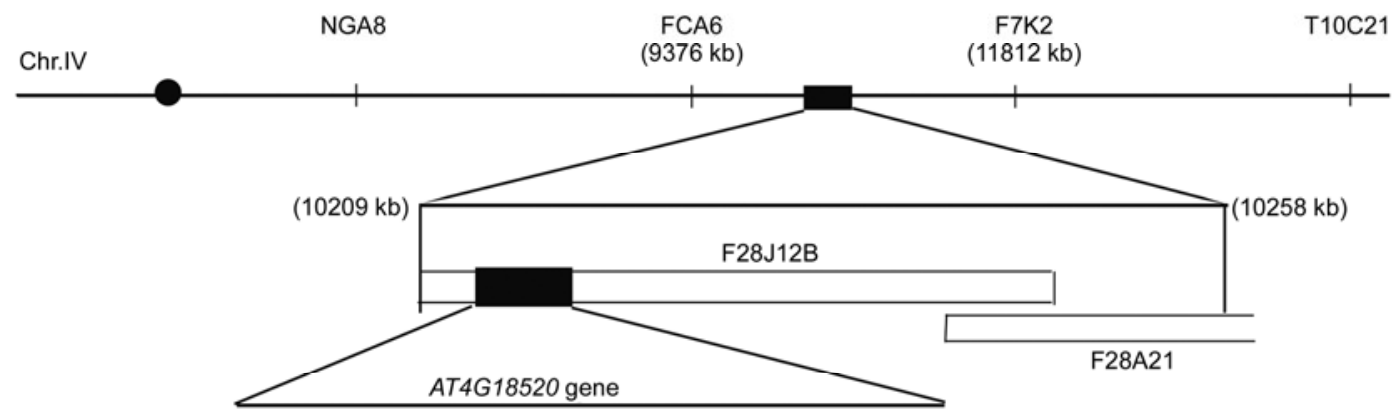

(c)

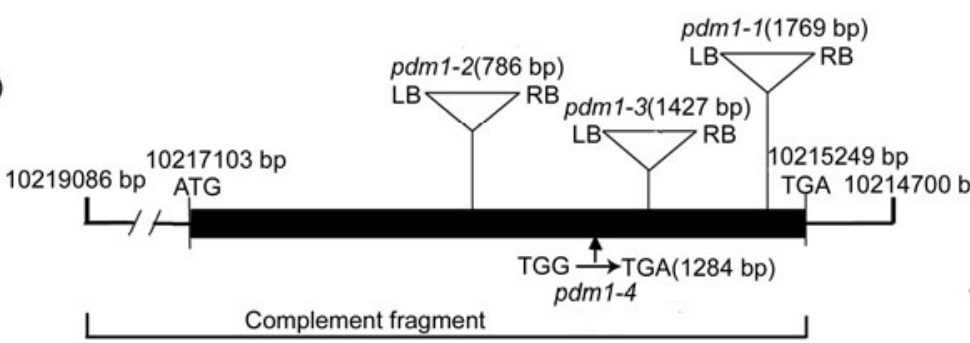

(d)

(d)

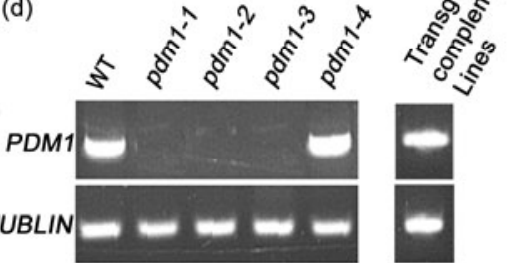

Figure 1 Characterization of the $p d m 1-1$ mutant phenotype and the $P D M 1$ gene. (a) Phenotypes of the wild-type Columbia-0(WT), $p d m 1-1, p d m 1-2$, pdm1-3 and pdm1-4 mutants, and the complemented pdm1. (b) Mapping of the PDM1 Locus. Black spot, the centromere. Fine mapping of PDM1 to a 49.6-kb region between the In/Del markers F28J12 and F28A21 on chromosome 4. (c) The PDM1 gene structure and T-DNA insertions of pdm1-1, pdm1-2 and $p d m 1-3$, and the position of the nucleotide change in pdm1-4. The complement fragment is indicated. (d) Expression analysis of the PDM1 gene in the WT and pdml mutants. TUBLIN expression is indicated as a control.

of the chloroplasts in 14-d-old leaves were examined under transmission electron microscopy (TEM). The mature WT chloroplasts exhibited normal stacked grana thylakoids, stroma thylakoids and starch grains. In contrast, only a few unstacked thylakoid lamella without stacked grana thylakoids were observed in the $p d m 1-1$ mutant (Figure 2). These results demonstrate that $P D M 1$ is essential for chloroplast development.

\subsection{PDM1 encodes a PPR protein localized to chloroplast}

Arabidopsis PDMI encodes a PPR protein harbouring 14 PPR motifs, which are arranged as S-P-L-S-P-L-S-P-L-S$\mathrm{P}-\mathrm{L}-\mathrm{S}-\mathrm{P}$. In the $\mathrm{C}$ terminus of the PDM1 protein, there are extra 32 amino acids. However, this sequence is different from the currently identified E, E+ and DYW domains. Thus, PDM1 is classified into the PLS subgroup. In the pdm1-1 mutant, the last 28 amino acids are deleted, suggesting this region is essential for PDM1 function (Figure 3(a)). Homologues of the PDM1 protein have been identified
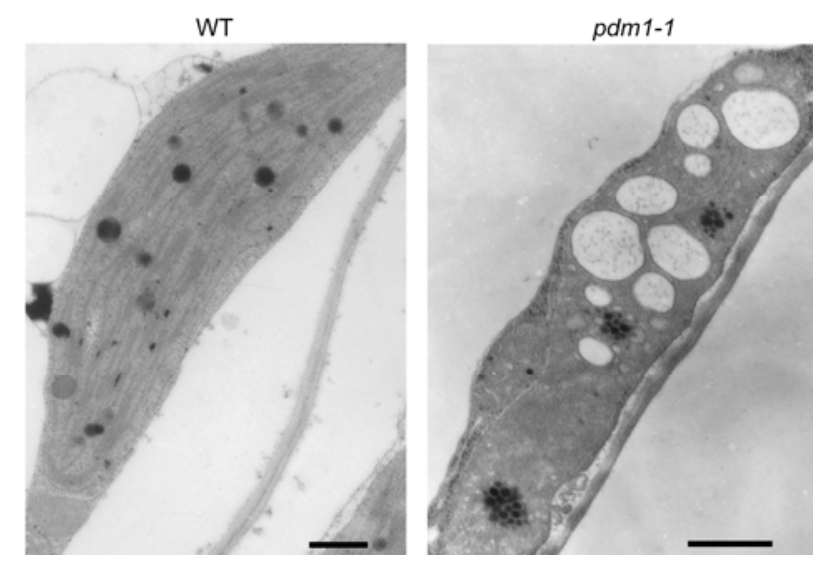

Figure 2 Ultrastructure of chloroplasts in the wild-type and $p d m 1-1$. In the mature chloroplasts of the WT, the stacked grana thylakoids and nonstacked stroma thylakoids are well organized, but in the pdm1-1 mutant the early development of chloroplasts has been disturbed. Scale bars $=0.5 \mu \mathrm{m}$.

in various species by BLASTp or tBLASTn search in the NCBI database. Those proteins sharing a high degree of 


$P$

MFSLSLIQPRLRISEIPVTQSYKSPTICYSSDSRTKREEQRHVRLPGFRLVSGKRASFDSGFSGFKGENVNQDDSSSFDSERVDYALLAEWLQSSNGMRLIKRIHAMALKCFDDQ VIYFGNNLISSCVRLGDLVYARKVFDSMPEK NTVTWTAMIDGYLKYGLEDEAFALFEDYVKHGIRF NLIVESSLVYFYAQCGELTSALRAFDMMEEK DVISWTAVISACSRKGHGIKAIGMFIGMLNHWFLP DVFVGTSLMDMYAKCGEISDCRKVFDGMSNR NTVTWTSIIAAHAREGFGEEAISLFRIMKRRHLIA NVYIGSTLVWLYCKCGESRDAFNVLQQLPSR DVVSWTAMISGCSSLGHESEALDFLKEMIQEGVEP NVFVGSALIHMYAKCGFVSEAFRVFDSMPEK

(b)

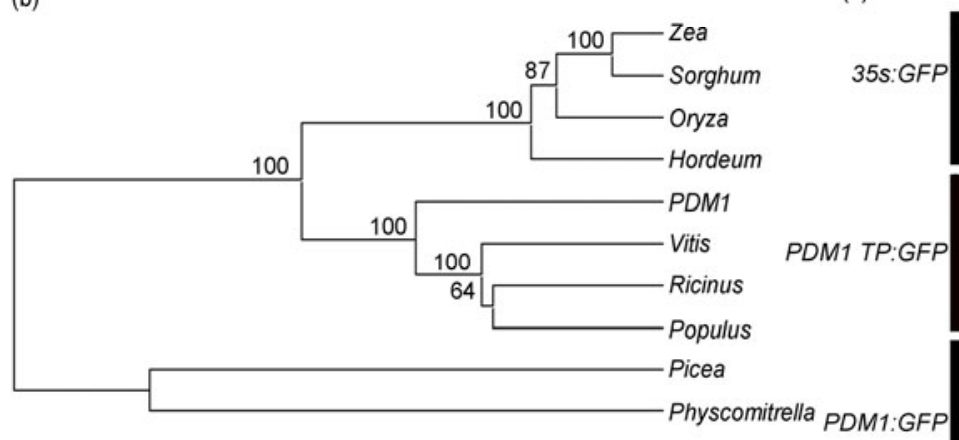

TNERMFVCLLNLCSRRAEFELGRQVHGNMVKVGVG NEFTVCSILKACSEEKALRFGRQVHSLVVKRMIKT NNLTVVSILRACGSVGALLLGKELHAQIIKNSIEK NPFTYSSALKACANSESLLIGRSIHSIAKKNHALS DDYIFATILSTCGDIELDEAVESSATCYLETS

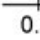

0.5

0.4

0.3

0.2

0.1

0.0

Chlorophyll

GFP
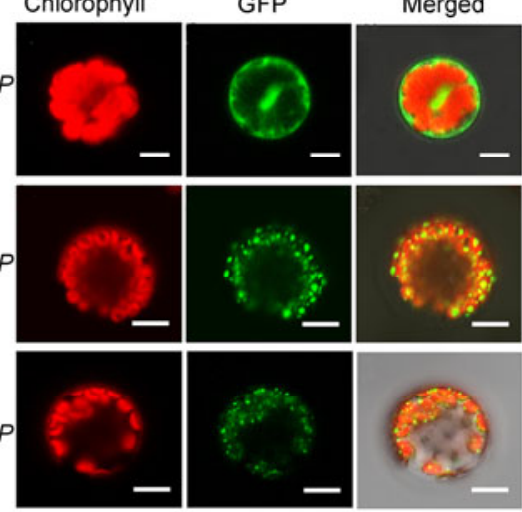

Figure 3 Phylogenetic analysis of PDM1 homologous proteins and subcellular localization of the PDM1 protein. (a) Protein structure of PDM1. The transit peptide is boxed. Black arrows indicate T-DNA interruption in the pdm1-1 mutant. (b) Phylogenetic tree of PDM1 and homologous proteins. Protein sequence files: Vitis vinifera, XP_002284293.1; Ricinus communis, XP_002513855.1; Populus trichocarpa, XP_002307403.1; Hordeum vulgare, BAJ99605.1; Oryza sativa, NP_001173562.1; Sorghum_bicolor, XP_002464828.1; Zea mays, ACG29369.1; Picea sitchensis, ABR17838.1; Physcomitrella, PpPPR_77. The amino acid sequences of the PDM1 homologous proteins were analyzed by the UPGMA method with the genetic distance calculated by Mega 3.1. (c) Subcellular localization of GFP fluorescence in the transformed protoplasts with the constructs of PDM1:GFP and PDM1 TP:GFP. Green fluorescence indicates the localization of PDM1:GFP and PDM1 TP:GFP proteins, while the red fluorescence is the autofluorescence of chlorophyll. Scale bars=20 $\mu \mathrm{m}$.

similarity with the PDM1 are used for phylogenetic analysis. In the phylogenetic tree PDM1 forms a basal clade within the dicotyledons (Figure 3(b)). The results indicated that PDM1 has been conserved in the evolutionary process, and that putative homologues are present in various land plants, including mosses, gymnosperm and angiosperm.

TargetP program analysis showed that the 19 amino acids at the N-terminus of PDM1 had the capacity to function as a plastid transit peptide (http://www.cbs.dtu.dk/services/ TargetP) [19]. To investigate this possibility, we fused the first 67 amino acids and the full-length PDM1 protein to the $\mathrm{N}$-terminus of a green fluorescent protein (GFP) to obtain PDM1 TP:GFP and PDM1:GFP constructs. These constructs, driven by a cauliflower mosaic virus (CaMV) $35 \mathrm{~S}$ promoter, were transformed into Arabidopsis protoplasts. At the same time, the only GFP driven by the $35 \mathrm{~S}$ promoter was transformed into protoplasts as a control. In the protoplasts transformed with the control construct, the GFP fluorescence was observed in the cytoplasm. However, in the protoplasts transformed with the PDMI TP:GFP construct or PDM1:GFP construct, the GFP fluorescence co-localized with the red autofluorescence of chlorophyll (Figure 3(c)). These results confirm that the Arabidopsis PDM1 protein is localized to the chloroplast.

\subsection{The defective processing of the $R p o A$ polycistronic transcript leads to a low accumulation level of the RpoA protein}

It was reported that monocistronic rpoA transcript cannot be detectable and that PEP-dependent chloroplast gene expression is affected in the $p d m 1-1$ mutant [12]. In the $p d m 1-1$ mutant, the $990 \mathrm{nt}$ transcript for rpoA was lacking, while two new transcripts $\mathrm{S} 1$ and S2 were processed. The transcript $\mathrm{S} 1$ is shorter than the dicistronic transcript $S 11$-rpoA. The transcript S2 is longer than the S11-rpoA dicistronic transcript, but shorter than the L36-S11-rpoA tricistronic transcript (Figure 4(a)).

To investigate whether the translation of RpoA was affected, a polyclonal antibody against RpoA was prepared in rabbits. A peptide specific for RpoA was used for immunization. The specific anti-RpoA antibody was purified, and then the RpoA antibody was tested for activity and specificity using recombinant protein (Figure 4(b)). This verified antibody was used to analyze the accumulation of RpoA protein in the pdm1-1 mutant and the WT. The result showed that the amount of RpoA in the pdml-1 mutant is approximately $1 / 3$ the level in the WT (Figure 4(c)). These results indicate that the defective rpoA polycistronic processing affects the 
(a)

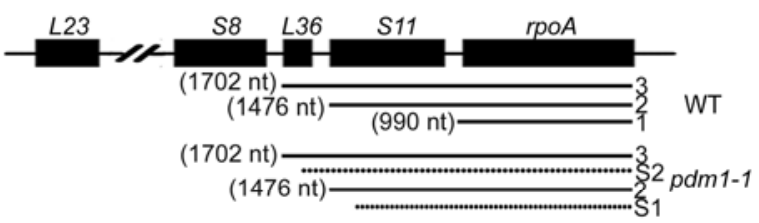

(b)

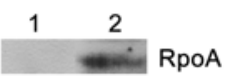

(c)

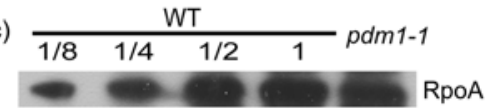

Figure 4 Schematic diagram of the rpoA transcripts and the change in RpoA protein accumulation in the pdm1-1 mutant. (a) Schematic diagram of smaller transcripts containing $r p o A$ according to Wu et al. [12]. Black lines, normal transcripts in the WT and the pdm1-1 mutant; dashed lines, additional transcripts in the pdm1-1 mutant. (b) Activity and specificity identification of anti-RpoA antibody. The rpoA gene from Arabidopsis recombined with pET-51b vector was introduced into $E$. coli Rosseta strain. 1, Lysate of Rosseta expressing pET-51b; 2, lysate of Rosseta expressing pET-51b-RpoA. (c) RpoA protein accumulation characterization. Total proteins were extracted from 2-week-old WT and pdm1-1 mutant plants, respectively, then size-fractionated by SDS-PAGE, transferred to a PVDF membrane, and detected with anti-RpoA antibodies.

translation and accumulation of the RpoA protein in the pdml-1 mutant.

\subsection{PDM1 is associated with the rpoA polycistronic transcripts}

PPR motifs have been demonstrated to have the capacity to bind RNA transcripts in vivo $[6,8,20,21]$. To determine whether PDM1 associate with rpoA pre-mRNA, RNA immunoprecipitation (RIP) was performed. In the complementation experiment, we obtained a transgenic Arabidopsis line that expresses PDM1 fused to the FLAG tag which can rescue the albino phenotype (Figure 1(a)). Expression of the fusion protein was verified by Western blot assay with an anti-FLAG M2 monoclonal antibody (Figure 5(a)). This transgenic line was used for RIP analysis. The PDM1FLAG protein was immunoprecipitated from the crude chloroplast protein extract of the transgenic plants using Dynabeads decorated with anti-FLAG antibodies (IP+). As a negative control (IP-), Dynabeads without antibodies were used. Then RNA extracted from the immunoprecipitated products was used for quantitative real-time RT-PCR analysis. When the primer pairs for the intergenic region of S11-rpoA (designated RB1) and the L23-L2.1 (designated RB7) were used (Figure 5(b)), the copy number of the PCR products from the anti-FLAG precipitate was nearly 88 -fold and 14-fold that of those from the mock immunoprecipitation respectively (Figure 5(c)). To further understand the binding of PDM1 with rpoA pre-mRNA, we quantified the RNA level of the fragments RB2, RB3, RB4, RB5 and RB6 in the anti-FLAG precipitate (Figure 5(b)). The quantitative real-time RT-PCR results showed that RB2 in the antiFLAG immunoprecipitated samples had a similar enrichment level as RB1, being nearly 88 -fold that of mock (a)

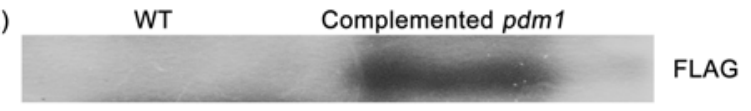

(b)

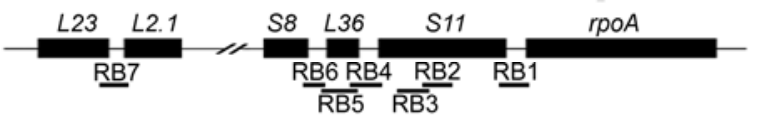

(c)

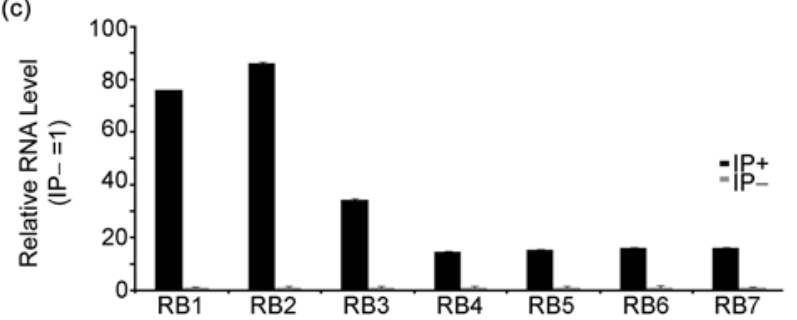

Figure 5 RNA immunoprecipitation analysis of PDM1 and associated RNA. (a) Verification of PDM1-FLAG protein accumulation. Total proteins were extracted respectively from 2-week-old WT and complemented PDM1-FLAG transgenic plants, then immunoblot analysis was performed with anti-FLAG antibodies. (b) Distribution of the gene fragments in the polycistron L23-L2-S19-L22-S3-L16-L14-S8-L36-S11-rpoA for RNA immunoprecipitation analysis. (c) RNA immunoprecipitation analysis. Chloroplasts fixed with FA were extracted from the PDM1-FLAG transgenic plants, and then all of the RNA transcripts were sonicated into short fragments under specific conditions and incubated with a Dynabeads-linked FLAG antibody. With the interaction between FLAG antibody and PDM1FLAG protein, associated RNA was immunoprecipitated and analyzed through realtime RT-PCR. IP+, anti-FLAG immunoprecipitation; IP-, mock immunoprecipitation. Data presented are mean values obtained from three replicates. Bars denote standard errors.

immunoprecipitation. However, RB3, RB4, RB5 and RB6 exhibited much lower enrichment (Figure 5(c)). These results suggest that PDM1 can bind to the 511 -rpoA intergenic sequence.

\section{Discussion}

In this study, the function of the PPR protein PDM1 in RNA processing and chloroplast development was investigated in Arabidopsis. A defect in rpoA processing resulted in a low level of accumulation of RpoA protein, which exerted an effect on the expression of PEP-dependent plastid genes and chloroplast development in the knockout mutant pdm1-1. The RNA immunoprecipitation results suggest that PDM1 binds to the intergenic sequence between $r p o A$ and $S 11$ to carry out endonucleolytic cleavage of rpoA from the precursor polycistronic transcripts.

\subsection{PDM1 is essential for chloroplast development in Arabidopsis thaliana}

Many factors have been reported to influence chloroplast development, including ribosome assembly [22], thylakoid membrane formation [23] and chloroplast gene expression [24]. In chloroplast development, PEP is indispensable for 
chloroplast gene expression as part of the essential transcription machinery. In recent years, many proteins have been reported to regulate PEP activity, with most of them belonging to the plastid Transcriptionally Active Chromosome (pTAC) complex. The knockout of these genes affects PEP activity and chloroplast development $[25,26]$. According to the results presented here, the pdml-1 mutant displays an albino phenotype with severely defective chloroplast development (Figure 2), while the results of Wu et al. [12] reveals that PEP-dependent gene expression is dramatically reduced in this mutant. These suggest that the defective PEP activity in $p d m l$ is the major factor that results in its lethal albino phenotype.

RpoA is a core subunit of PEP. The normal $990 \mathrm{nt} r p o A$ transcript is cleaved from polycistronic transcripts and is responsible for efficient RpoA protein synthesis in the WT. In this work, our results show that PDM1 is associated with the rpoA polycistronic transcript (Figure 5). However, a new transcript of approximate $1300 \mathrm{nt}$ (S1, Figure 4(a)) was present in the $p d m 1-1$ mutant instead of the $990 \mathrm{nt}$ transcript observed in the WT [12]. The RpoA protein can be translated with low efficiency and its accumulation amounted to about $1 / 3$ of the WT (Figure 4). This suggests other transcripts of rpoA may be used as templates for protein translation. This decreased RpoA amount in pdml would be expected to exert an effect on PEP activity. However, PEP seems to have very low activity in $p d m 1-1$ mutant [12]. The PPR proteins have a range of essential functions in posttranscriptional processes [3]. For instance, PPR10 can stimulate atpH translation in addition to stabilizing the $5^{\prime}$ and $3^{\prime}$ chloroplast mRNA termini [8]. Additionally, the PPR protein CRP1 is required for the accumulation of processed transcripts of pet $B$ and pet $D$ in maize chloroplasts and the translation of RNAs $[11,20]$. Given the complexity of the functions of the PPR proteins, the possibility cannot be ruled out that PDM1 may be involved in an as yet unidentified process which affects PEP activity.

\subsection{PDM1 is involved in the endonucleolytic cleavage of $S 11$ and rpoA}

PDMI encodes a PPR protein of the PLS subfamily (Figure 3). PPR motifs have been demonstrated to have a capacity to bind RNA and the PDM1 protein consists of 14 PPR motifs. Since the RIP result showed that PDM1 was associated with the S11-rpoA intergenic sequence, PDM1 may bind to rpoA pre-mRNA. In the $\mathrm{C}$ terminus of PDM1, there is an extra 32 amino acid sequence that does not belong to PPR motif. In the pdml-1 mutant, 28 of the 32 amino acids are deleted, and two new transcripts are processed instead of the $990 \mathrm{nt}$ transcript for rpoA (Figure 4(a)). This cleavage pattern change suggests that the $\mathrm{C}$ terminal region in PDM1 is responsible for the recognition of the PDM1 or PDM1 complex on the polycistronic precursor. However, there are no reports to show that PPR proteins have catalytic activity.
PDM1 might associate or interact with other proteins in order to perform the cleavage function. The amino acid sequence from $\mathrm{Val}^{116}$ to $\mathrm{Val}^{585}$ in PDM1 composes tandem arrays of PPR motifs. In the $\mathrm{N}$ terminus of the PDM1 protein, the first 19 amino acid sequence is predicted to be a transit peptide (Figure 3(a)). The amino acid sequence from $\mathrm{Gln}^{20}$ to $\mathrm{Gln}^{115}$ may play a role in the interaction with other proteins. The identification of proteins interacting with PDM1 should facilitate our understanding of the molecular mechanism that PDM1 regulate rpoA monocistronic RNA cleavage.

We thank ABRC Bioresources, which kindly offered the transgenic Arabidopsis lines (SALK_034701, CS835488 and SALK_005288). We would like to thank Nai-Ying Yang from SHNU for her skillful technical assistance in transmission electron microscopy. This work was supported by the National Natural Science Foundation of China (31100180).

1 Raven J A, Allen J F. Genomics and chloroplast evolution: What did cyanobacteria do for plants? Genome Biol, 2003, 4: 209

2 Sugiura M. The chloroplast chromosomes in land plants. Annu Rev Cel Biol, 1989, 5: 51-70

3 Lurin C, Andrés C, Aubourg S. et al. Genome-wide analysis of Arabidopsis pentatricopeptide repeat proteins reveals their essential role in organelle biogenesis. Plant Cell, 2004, 16: 2089-2103

4 de Longevialle A F, Hendrickson L, Taylor N L, et al. The pentatricopeptide repeat gene OTP51 with two LAGLIDADG motifs is required for the cis-splicing of plastid ycf3 intron 2 in Arabidopsis thaliana. Plant J, 2008, 56: 157-168

5 Meierhoff K, Felder S, Nakamura T, et al. HCF152, an Arabidopsis RNA binding pentatricopeptide repeat protein involved in the processing of chloroplast psbB-psbT-psbHpetB-petD RNAs. Plant Cell, 2003, 15: 1480-1495

6 Schmitz-Linneweber C, Williams-Carrier R E, Williams-Voelker P $\mathrm{M}$, et al. A pentatricopeptide repeat protein facilitates the transsplicing of the maize chloroplast rps 12 pre-mRNA. Plant Cell, 2006, 18: 2650-2663

7 Fisk D G, Walker M B, Barkan A. Molecular cloning of the maize gene $\operatorname{crp} 1$ reveals similarity between regulators of mitochondrial and chloroplast gene expression. EMBO J, 1999, 18: 2621-2630

8 Pfalz J, Bayraktar O A, Prikryl J, et al. Site-specific binding of a PPR protein defines and stabilizes $5^{\prime}$ and $3^{\prime}$ mRNA termini in chloroplasts. EMBO J, 2009, 28: 2042-2052

9 Hammani K, Okuda K, Tanz S K, et al. A study of new Arabidopsis chloroplast RNA editing mutants reveals general features of editing factors and their target sites. Plant Cell, 2009, 21: 3686-3699

10 Hashimoto M, Endo T, Peltier G, et al. A nucleus-encoded factor, CRR2, is essential for the expression of chloroplast ndhB in Arabidopsis. Plant J, 2003, 30: 541-549

11 Barkan A, Walker M, Nolasco M, et al. A nuclear mutation in maize blocks the processing and translation of several chloroplast mRNAs and provides evidence for the differential translation of alternative mRNA forms. EMBO J, 1994, 13: 3170-3181

$12 \mathrm{Wu} \mathrm{H}$, Zhang $\mathrm{L} \mathrm{X}$. The PPR protein PDM1 is involved in the processing of rpoA pre-mRNA in Arabidopsis thaliana. Chin Sci Bull, 2010, 55: 3485-3489

13 Motohashi R, Nagata N, Ito T, et al. An essential role of a TatC homologue of a $\Delta \mathrm{pH}$-dependent protein transporter in thylakoid membrane formation during chloroplast development in Arabidopsis thaliana. Proc Natl Acad Sci USA, 2001, 98: 10499-10504

14 Cui Y L, Jia Q S, Yin Q Q, et al. The GDC1 gene encodes a novel ankyrin domain containing protein that is essential for grana formation in Arabidopsis thaliana. Plant Physiol, 2011, 155: 130-141

15 Ketcham S R, Davenport J W, Warncke K, et al. Role of the $\gamma$ subunit of chloroplast coupling factor 1 in the light-dependent activation of 
photophosphorylation and ATPase activity by dithiothreitol. J Biol Chem, 1984, 259: 7286-7293

16 Terzi1 L C, Simpson G G. Arabidopsis RNA immunoprecipitation. Plant J, 2009 59: 163-168

17 Cao Z L, Yu Q B, Sun Y, et al. A Point Mutation in the pentatricopeptide repeat Motif of the AtECB2 protein causes delayed chloroplast development. J Integr Plant Biol, 2011, 53: 258-269

18 Zhang Z B, Zhu J, Gao J F, et al. Transcription factor AtMYB103 is required for anther development by regulating tapetum development, callose dissolution and exine formation in Arabidopsis. Plant J, 2007, 52: $528-538$

19 Emanuelsson O, Nielsen H, Brunak S, et al. Predicting subcellular localization of proteins based on their N-terminal amino acid sequence. J Mol Biol, 2000, 300: 1005-1016

20 Schmitz-Linneweber C, Williams-Carrier R, Barkan A. RNA immunoprecipitation and microarray analysis show a chloroplast pentatricopeptide repeat protein to be associated with the $5^{\prime}$ region of mRNAs whose translation it activates. Plant Cell, 2005, 17: 27912804

21 Beick S, Schmitz-Linneweber C, Williams-Carrier R, et al. The pen- tatricopeptide repeat protein PPR5 stabilizes a specific tRNA precursor in maize chloroplasts. Mol Cell Biol, 2008, 28: 5337-5347

22 Schultes E A, Bartel D P. One sequence, two ribozymes: Implications for the emergence of new ribozyme folds. Science, 2000, 289: 448452

23 Kobayashi K, Kondo M, Fukuda H, et al. Galactolipid synthesis in chloroplast inner envelope is essential for proper thylakoid biogenesis, photosynthesis, and embryogenesis. Proc Natl Acad Sci USA, 2007, 104: 17216-17221

24 Hajdukiewicz P T, Allison L A, Maliga P. The two RNA polymerases encoded by the nuclear and the plastid compartments transcribe distinct groups of genes in tobacco plastids. EMBO J, 1997, 16: 4041-4048

25 Pfalz J, Liere K, Kandlbinder A, et al. pTAC2, -6, and -12 are components of the Transcriptionally Active Plastid Chromosome that are required for plastid gene expression. Plant Cell, 2006, 18: 176-197

26 Gao Z P, Yu Q B, Zhao T T, et al. Functional component of the transcriptionally active chromosome complex, Arabidopsis pTAC14, interacts with pTAC12/HEMERA and regulates plastid gene expression. Plant Physiol, 2011, 157: 1733-1745

Open Access This article is distributed under the terms of the Creative Commons Attribution License which permits any use, distribution, and reproduction in any medium, provided the original author(s) and source are credited.

\section{Supporting Information}

Table S1 Primers for RNA immunoprecipitation analysis

The supporting information is available online at csb.scichina.com and www.springerlink.com. The supporting materials are published as submitted, without typesetting or editing. The responsibility for scientific accuracy and content remains entirely with the authors. 